\title{
Adhesion of Chlamydomonas microalgae to surfaces is switchable by light
}

\author{
Christian Titus Kreis, Marine Le Blay, Christine Linne, Marcin Michal Makowski \\ and Oliver Bäumchen ${ }^{\star}$
}

\begin{abstract}
Microalgae are photoactive microbes that live in liquid-infused environments, such as soil, temporary pools and rocks, where they encounter and colonize a plethora of surfaces. Their photoactivity manifests itself in a variety of processes, including light-directed motility (phototaxis) ${ }^{1-3}$, the growth of microalgal populations ${ }^{4}$, and their photosynthetic machinery ${ }^{5}$. Although microbial responses to light have been widely recognized, any influence of light on cell-surface interactions remains elusive. Here, we reveal that the unspecific adhesion of microalgae to surfaces can be reversibly switched on and off by light. Using a micropipette force spectroscopy technique, we measured in vivo single-cell adhesion forces and show that the microalga's flagella provide light-switchable adhesive contacts with the surface. This light-induced adhesion to surfaces is an active and completely reversible process that occurs on a timescale of seconds. Our results suggest that light-switchable adhesiveness is a natural functionality of microalgae to regulate the transition between the planktonic and the surface-associated state, which yields an adhesive adaptation to optimize the photosynthetic efficiency in conjunction with phototaxis.
\end{abstract}

Bioadhesion is a ubiquitous phenomenon in nature, as evidenced through a variety of adhesion strategies that different species have developed in adaptation to their natural habitat ${ }^{6-9}$. For microbial life, including bacteria and microalgae, surface association is ultimately linked to the formation of dense populations called biofilms, which have profound implications in natural, physiological and technological settings ${ }^{10,11}$. Microalgae are representatives of photoactive microbes and inherit abundant ecological, environmental and technological relevance, for example, in water purification systems, bioremediation, and photo-bioreactors for the sustainable production of biofuels ${ }^{12,13}$.

For these motile microorganisms, it was recognized that their flagella represent an effective tool for providing contact and cell adhesion to surfaces, in addition to realizing cellular motility ${ }^{14,15}$. Although there have been significant advancements towards a fundamental understanding of bacterial and cellular adhesion ${ }^{16}$, in vivo single-cell adhesion measurements of motile microalgae are lacking due to limitations of the conventional techniques. Hence, the adhesion forces and the mechanisms that trigger flagella-mediated cell adhesion to surfaces remain elusive.

The biflagellated microalga Chlamydomonas is a model organism in cellular biology ${ }^{17}$ to study cell metabolism ${ }^{18}$, photosynthesis $^{5}$, phototaxis ${ }^{1-3}$ and light receptors ${ }^{19}$, while its flagella represent a model system for the eukaryotic microtubule-based axoneme ${ }^{15}$. In its planktonic state (freely swimming), Chlamydomonas swims with a breaststroke-like beating of its two flagella ${ }^{14}$, and performs phototaxis to optimize its photosynthetic efficiency ${ }^{1-3}$. Although the swimming motility of Chlamydomonas has been widely studied in recent years, the cell's natural habitats include complex confining environments, such as soil and temporary pools. A cell's association to surfaces is mediated by flagella-surface contacts, which are the basis for surface gliding as an alternative motility mode ${ }^{15}$. Gliding is realized by coupling the flagella adhesive sites, the membrane glycoprotein FMG-1B ${ }^{20}$, via intraflagellar transport trains to molecular motors that move along the microtubules ${ }^{21-24}$. The forces exerted by a single motility domain are on the order of several tens of piconewtons, as inferred from optical tweezers experiments $^{22,23}$. The study of flagella adhesion has so far been limited to isolated flagella in ex situ atomic force microscopy measurements ${ }^{25}$, which cannot capture any active response of living Chlamydomonas cells.

Here, we show in vivo force spectroscopy experiments that allow for a quantitative analysis of the flagella-mediated adhesion of Chlamydomonas to substrates, yielding three main results. First, the adhesion of Chlamydomonas can be reversibly switched on and off by light. Second, the transition between the planktonic and the surface-associated state in response to light stimulation occurs on a timescale of seconds. Third, our experiments suggest that the light-switchable adhesion is due to the redistribution of adhesionpromoting flagella membrane proteins, which enables the cell to adapt the adhesiveness of its flagella.

We report on single-cell experiments with vegetative wild-type Chlamydomonas reinhardtii cells in aqueous environment under precisely controlled light conditions (see Methods). Micropipette force sensors ${ }^{26}$ were employed for grasping a living cell in a manually controlled orientation and bringing the cell into contact with a substrate (see Fig. 1a,b). By moving the substrate, we obtained adhesion forces from force spectroscopy experiments by recording the micropipette deflection during reiterative approach and retraction cycles (see Supplementary Movies 1 and 2). Force-distance curves for cells in opposite orientations provide evidence that solely the flagella of the microalga adhere to the substrate (see Fig. 1c), whereas the cell body does not exhibit any detectable adhesion force (see Supplementary Fig. 1). In the following, we exclusively report on adhesion forces mediated by the Chlamydomonas flagella (see configuration depicted in Fig. 1c). The adhesion forces of different cells are typically in the range from 1 to $4 \mathrm{nN}$ (mean force: $3.7 \mathrm{nN}$, median force: $2.8 \mathrm{nN}$ ), but can be as large as $15 \mathrm{nN}$ in rare instances (see inset Fig. 1c). The broadness of the distribution can be attributed to some cell-cell variability, as shown in Supplementary Fig. 2.

We demonstrate that the flagella adhesiveness can be reversibly switched on and off by changing the illumination from white to 
a

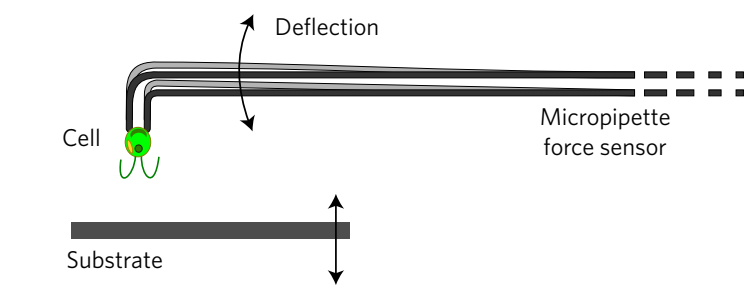

b

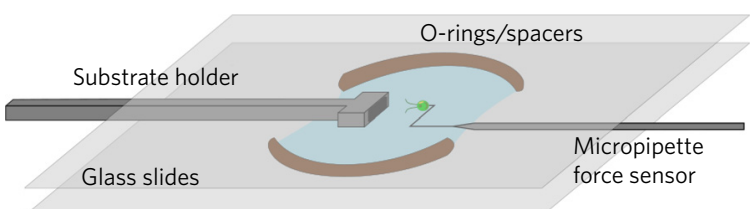

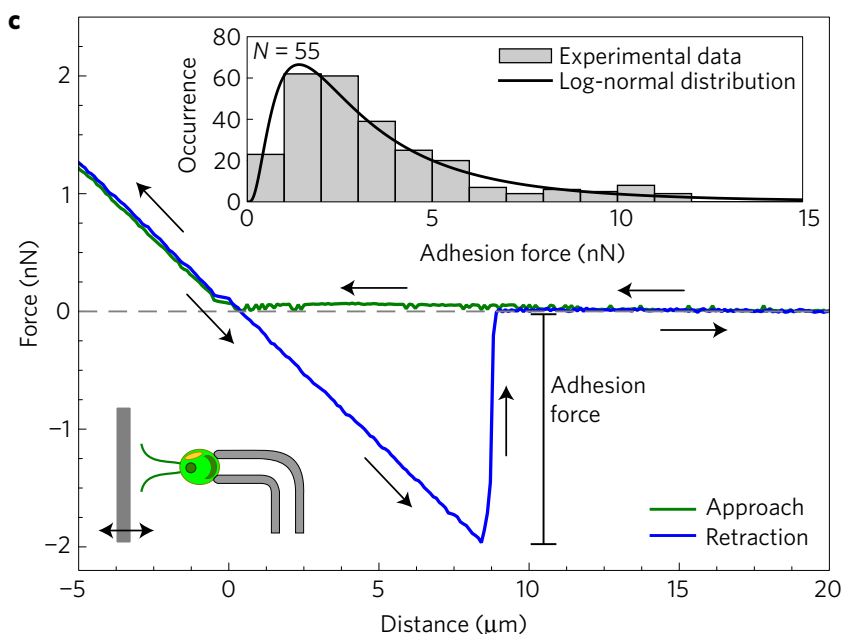

Figure 1 | In vivo micropipette force spectroscopy of Chlamydomonas reinhardtii microalgae. a, Measurement principle: a living cell is held with a micropipette, which deflects upon interaction of the cell with a moving substrate. $\mathbf{b}$, Experimental set-up: a liquid cell is designed such that a double-L-shaped micropipette and a substrate holder can enter from opposite sides. c, Force-distance curves (see Methods) provide evidence that the flagella adhere to the substrate. The adhesion force is given as the force required to detach the cell from the substrate. The displayed curve represents raw data from a single run without any averaging. The inset depicts the statistical distribution of adhesion forces (mean: $3.7 \mathrm{nN}$, median: $2.8 \mathrm{nN}$ ) recorded for 55 cells (see Supplementary Fig. 2). The solid line represents a log-normal distribution (see Methods).

red light. This finding is evidenced by a series of consecutive force-distance curves in white light (see Supplementary Movie 1) and red light (see Supplementary Movie 2) at constant illumination intensity (see Fig. 2a), which we repeatedly recorded for the same cell (see Fig. 2b). Comparing the same cells in different light conditions, we found that, in $95.2 \%$ of all experiments, the adhesion forces in red light were smaller than one standard deviation of the average adhesion force in white light. This includes $72.4 \%$ of all measurements in red-light conditions without any detectable adhesion (typical noise level of approx. 20-30 pN), whereas $100 \%$ of the force-distance curves exhibited an adhesion peak in white light (see inset Fig. 2a). The illumination intensity (irradiance) did not show any influence on the adhesion forces in the tested range of $10^{19}-10^{21}$ photons $\mathrm{m}^{-2} \mathrm{~s}^{-1}$ (see Fig. $2 \mathrm{c}$ ).

The illumination wavelength plays a crucial role for the adhesion properties of the flagella: for the same cells exposed to different wavelengths at constant irradiance, we recorded an adhesion peak exclusively upon exposure to blue light (see Fig. 2d), which strongly suggests that a blue-light receptor controls the flagella adhesiveness in Chlamydomonas. The adhesion forces and their distribution for different cells in blue light were found to be consistent with the results obtained in white light (see Fig. 2e). These results are substantiated by adhesion experiments in which the bluelight intensity was gradually decreased. As shown in Fig. $2 \mathrm{f}$, we identified an irradiance threshold at $2-5 \times 10^{18}$ photons $\mathrm{m}^{-2} \mathrm{~s}^{-1}$, below which the flagella do not show any adhesiveness. This value is consistent with the sensitivity of photoreceptors that trigger other photoresponses in Chlamydomonas ${ }^{3,27}$. Potential candidates for the blue-light photoreceptor that triggers the flagella adhesiveness include channelrhodopsins, which are responsible for the phototactic behaviour in aqueous environments ${ }^{2,27}$, as well as phototropin, which can be found in the flagellum, controls the cell's life cycle and governs several aspects of the mating process ${ }^{17,28}$.

Gaining insight into the switchability of the flagellar adhesiveness and the transition from the planktonic to the surface-associated state demands further experimental enquiries. Complementary to the force spectroscopy, the micropipette technique allows for time-resolved force measurements. In this approach, the substrate position remained fixed and the Chlamydomonas cell was held at a distance of approximately $4-8 \mu \mathrm{m}$ to the substrate. Although the cell body is not in contact, the tips of the flagella can physically sense the substrate through contact interactions with the surface during every beating cycle (see Fig. 3a). We optically tracked the cell's response to external white illumination through the configuration of their flagella using high-speed imaging (see Fig. 3a) and, additionally, through the corresponding deflection of the micropipette (see Fig. 3b). Note that, during the measurement, red light was always on to detect the micropipette deflection during periods when white light was off. When the white illumination is turned on, the flagella adhered to the surface (see Supplementary Movie 3) and pulled the cell body towards the substrate (see Fig. 3a,b and Supplementary Movie 4). This active process, which we refer to as 'auto-adhesion', is terminated as soon as the cell has achieved a stationary configuration on the substrate, with its flagella being straight and oriented at $180^{\circ}$ from one another (termed gliding configuration, see Supplementary Fig. 8). After turning off the white illumination, the micropipette returned to the zero-force position as the cell detached from the substrate and the flagella recovered their breaststroke beating. The auto-attachment of the flagella and the cell's active approach towards the substrate are reversible, as seen by exposing the cell to alternating light conditions (see Fig. 3d). The characteristic timescales of these active processes were extracted from the optical micrographs together with the micropipette deflection signal. In high-speed and high-resolution optical micrographs (see Fig. 3a), we find that it takes a few seconds after the white light was turned on before one or both flagella tips adhere to the surface (on average $10.6 \pm 9.2 \mathrm{~s}$ for adhesive flagellasurface contacts that last longer than one full beating cycle). The immobilization of the tip of a flagellum, through its adhesion to the substrate, results in a drastic modulation of its beating pattern and, eventually, terminates the beating (see Supplementary Movie 3). The delay for the onset of the active approach was inferred from the micropipette deflection data (see Fig. 3b), yielding delay times of up to several tens of seconds (see Fig. 3c).

The cell's light response during swimming manifests itself through the phototactic behaviour, which is based on the adaptation of the flagella beating induced by channelrhodopsin-triggered photocurrents (characteristic timescales of tens of milliseconds) ${ }^{3,29}$. The timescales observed in our time-resolved force measurements on the adhesiveness of the flagella are substantially longer than the light adaptation of the flagella beating, although both effects might be triggered by the same photoreceptor. These results open 
a

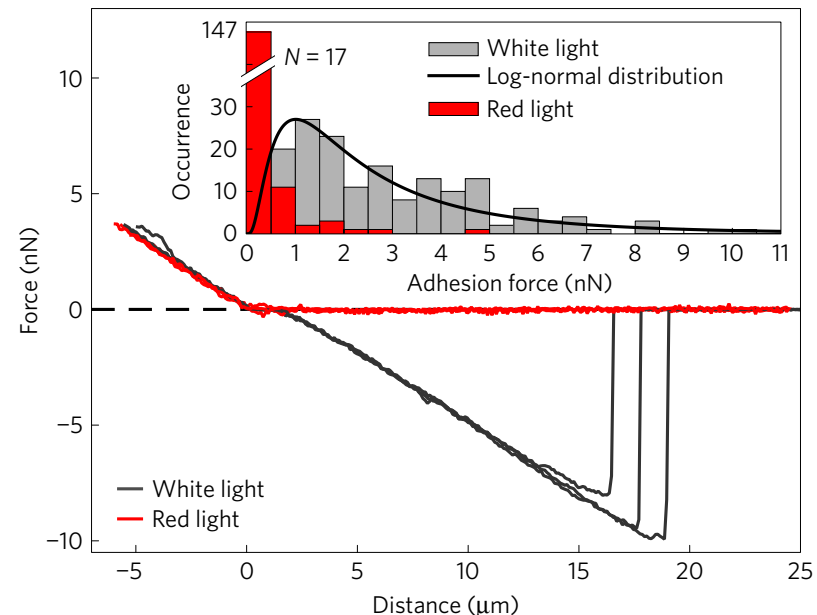

d

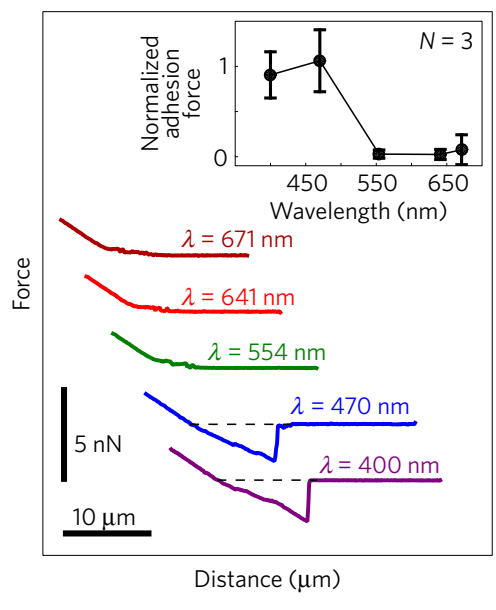

b
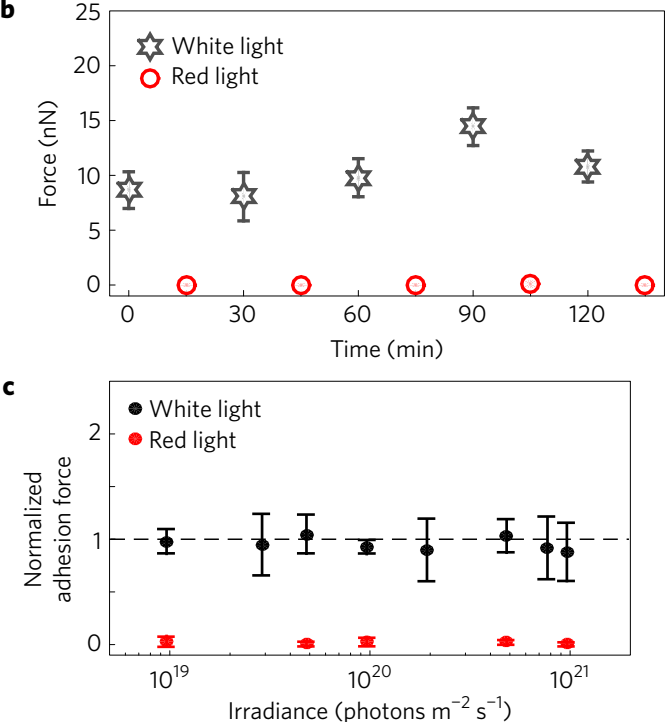

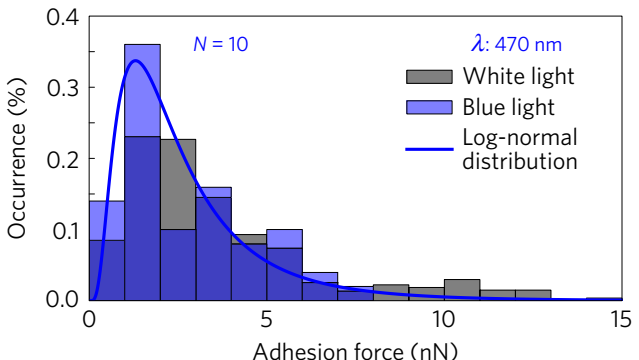

$\mathbf{f}$

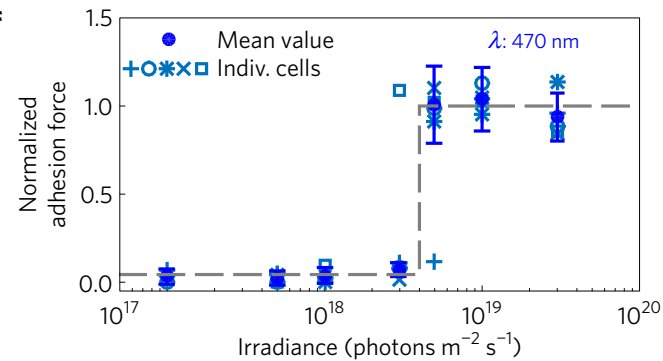

Figure $\mathbf{2}$ | In vivo micropipette force spectroscopy of Chlamydomonas in different light conditions. a, Force-distance curves (retraction) of the same cell in white (dark grey lines) and red light (red lines). (Inset) Distribution of adhesion forces for $N=17$ different cells in red and white light. $\mathbf{b}$, Adhesion force measurements for the same cell in alternating light conditions. c, Illumination intensity (for irradiances $\geq 10^{19}$ photons $\mathrm{m}^{-2} \mathrm{~s}^{-1}$ ) does not influence the adhesion force in white and red light. $\mathbf{d}$, Force-distance curves (retraction) for the same cell using different illumination wavelengths. Cells exclusively adhere in blue light. (Inset) Mean adhesion force of different cells $(\mathrm{N}=3)$ normalized to their adhesion force in blue light $(470 \mathrm{~nm})$. e, Distribution of adhesion forces for $N=10$ cells in blue $\left(470 \mathrm{~nm}\right.$ ) and white light (see Fig. 1c). $\mathbf{f}$, Identification of an intensity threshold at $2-5 \times 10^{18}$ photons $\mathrm{m}^{-2} \mathrm{~s}^{-1}$ in blue light $(470 \mathrm{~nm})$. Dashed line represents a guide to the eye. See Methods for details. Error bars represent standard deviations.

the fundamental question concerning the underlying processes on the molecular level at the flagella membrane.

To elucidate the mechanism of how the flagella can switch their adhesiveness, we tested the effect of proteolysis on the adhesion forces in controlled light conditions. In white illumination, the exposure to pronase resulted in a gradual decrease of the adhesion force towards zero adhesion (see Fig. 4a), caused by the digestion of adhesion-promoting proteins into individual amino acids. Note that pronase is not specific to a certain protein, but rather digests all proteins at the surface of the flagella membrane. In a reference experiment, the adhesion force remained constant in the absence of any pronase treatment during the same time frame (see Supplementary Fig. 3a). We applied the same pronase treatment to cells in red light for two hours and quantified their adhesion force immediately after turning on the white light. In contrast to the white-light proteolysis, these experiments showed that a pronase treatment in red light did not reduce the adhesion force to zero (see Fig. 4b). All cells still exhibited adhesiveness to the substrate after red-light proteolysis. The measured adhesion forces were slightly smaller as compared to the initial value, but consistent with independent adhesion force measurements performed immediately after turning on white light (see Supplementary Fig. 3b). These proteolysis results indicate that the adhesion-promoting proteins are not exposed at the surface of the flagella membrane in red light and, thus, a protein redistribution mechanism appears to be a possible explanation for the light-switchable flagella adhesiveness.

The adhesion of Chlamydomonas to surfaces and the lightswitchability effect are unspecific in the sense that both work independently of the substrate (see Supplementary Fig. 7 for a comparison between different substrates), in contrast to specific adhesion mechanisms that rely on, for example, ligand-receptor interactions. Taking together the findings that the reported adhesion phenomenon is unspecific on the one hand and protein-mediated on the other, we consider a dominant role of electrostatic interactions, potentially originating from net charges of the adhesion-mediating proteins. In fact, protein-mediated electrostatic interactions are responsible for the surface adhesion of various natural systems in aqueous environments-an example being the effective adhesive 
a

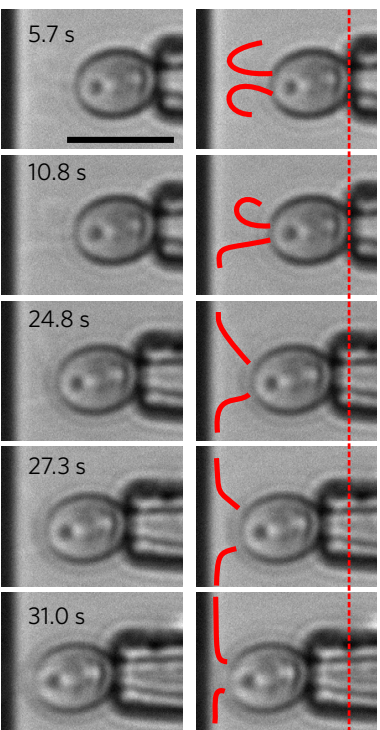

b

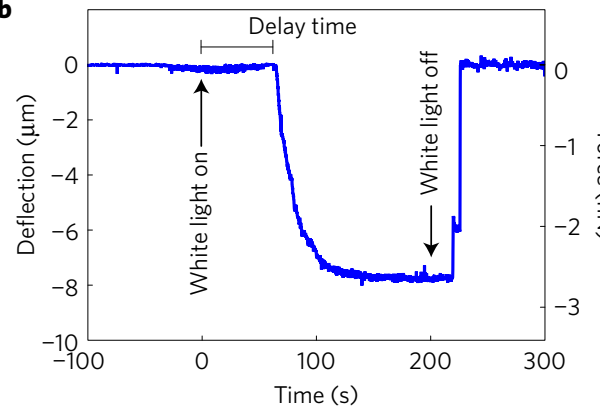

c

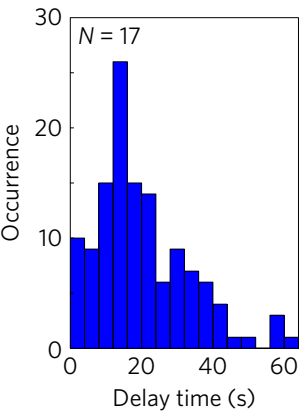

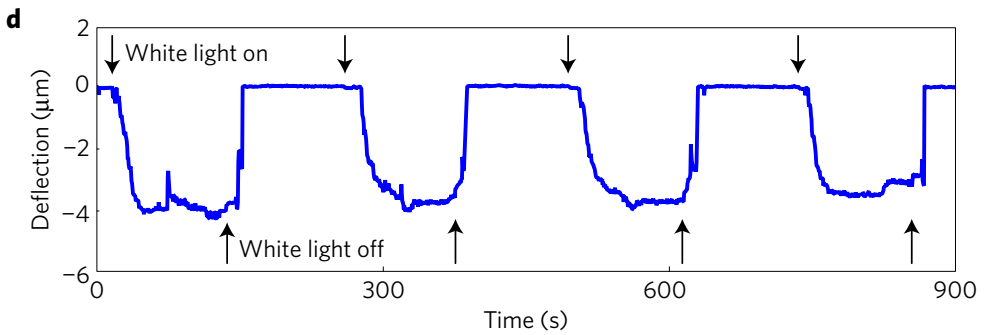

Figure 3 | Light-switchable auto-adhesion of Chlamydomonas. a, Optical micrographs of a cell held with a micropipette force sensor. The right-hand column includes guidelines to the eye (solid, flagella; dashed, initial micropipette position). The flagella actively pull the cell towards the substrate. Times given after white light was turned on. Scale bar, $10 \mu \mathrm{m}$. b. Micropipette deflection signal of the cell's auto-attachment and -detachment. c, Distribution of delay times between the onset of white illumination and the micropipette's response ( $N=17$ cells). $\mathbf{d}$, Micropipette deflection signal for a cell exposed to alternating illumination conditions.
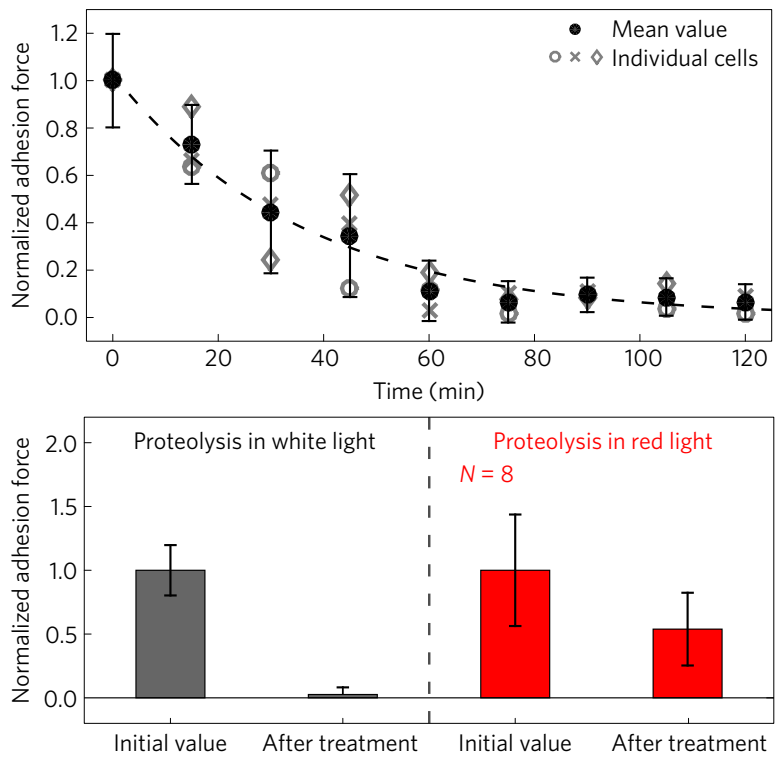

Figure 4 | Effect of proteolysis in different light conditions on the adhesiveness of Chlamydomonas. a, In white illumination the mean adhesion force decreases over time to less than $5 \%$ of the initial value before pronase treatment. Dashed line represents a guide to the eye. b, Comparison of the adhesion force before and after treatment (120 min) with pronase. Whereas the adhesiveness is lost after white-light proteolysis, cells still exhibit adhesiveness after red-light proteolysis $(N=8)$. Note that adhesion forces were measured in white light. Error bars represent standard deviations.

proteins secreted by mussels ${ }^{7,8}$. While the hypothesis regarding the underlying intermolecular interactions in Chlamydomonas adhesion remains to be tested, earlier immobilized iodination studies using planar substrates and microspheres did provide evidence that the flagellar membrane protein FMG-1B is responsible for the adhesion of the flagella to surfaces ${ }^{20,30}$. FMG-1B is uniformly distributed along the flagellar surface and it is presumed that their large, glycosylated ectodomains form the 16-18-nm-thick 'glycocalyx' layer at the flagellar surface ${ }^{17}$.

In Chlamydomonas microalgae, intraflagellar transport drives the movement of the adherent flagella membrane proteins and enables the flagella-mediated gliding motility ${ }^{21-24}$. Our results suggest that it is precisely this process that drives the auto-adhesion during which the flagella accommodate on the substrate after contact. Light-switchable flagella adhesiveness enables planktonic cells to trigger the reversible transition from the freely swimming to the surface-associated state, which can be readily observed for Chlamydomonas swimming in confined liquid environments (see Supplementary Movie 5). In fact, this functionality of the eukaryotic flagella might have evolved as a biological advantage for photoactive microorganisms that are exposed to surfaces and variable light conditions in their natural habitats.

In the context of technological applications, controlling the adhesion properties of photoactive microalgae could significantly contribute to enhancing the efficiency of photo-bioreactors by inhibiting colonization and biofilm formation at surfaces. In bioengineering settings, we anticipate that this functionality could inspire new pathways for designing surfaces exhibiting lightswitchable adhesion properties and could be employed for the uptake and release of cargo in targeted cargo delivery scenarios ${ }^{31}$.

\section{Methods}

Methods, including statements of data availability and any associated accession codes and references, are available in the online version of this paper.

Received 18 January 2017; accepted 15 August 2017; published online 25 September 2017

\section{References}

1. Foster, K. W. \& Smyth, R. D. Light antennas in phototactic algae. Microbiol. Rev. 44, 572-630 (1980).

2. Foster, K. W. et al. A rhodopsin is the functional photoreceptor for phototaxis in the unicellular eukaryote Chlamydomonas. Nature 311, 756-759 (1984).

3. Harz, H. \& Hegemann, P. Rhodopsin-regulated calcium currents in Chlamydomonas. Nature 351, 489-491 (1991). 
4. Carvalho, A. P., Silva, S. O., Baptista, J. M. \& Malcata, F. X. Light requirements in microalgal photobioreactors: an overview of biophotonic aspects. Appl. Microbiol. Biotechnol. 89, 1275-1288 (2011).

5. Raven, J. A. \& Girard-Bascou, J. Algae model systems and the elucidation of photosynthetic metabolism. J. Phycol. 37, 943-950 (2001).

6. Autumn, K. et al. Adhesive force of a single gecko foot-hair. Nature 205, 681-685 (2000).

7. Lee, H., Scherer, N. F. \& Messersmith, P. B. Single-molecule mechanics of mussel adhesion. Proc. Natl Acad. Sci. USA 103, 12999-13003 (2006).

8. Maier, G. P., Rapp, M. V., Waite, J. H., Israelachvili, J. N. \& Butler, A. Adaptive synergy between catechol and lysine promotes wet adhesion by surface salt displacement. Science 349, 628-632 (2015).

9. Brau, F., Lanterbecq, D., Zghikh, L.-N., Bels, V. \& Damman, P. Dynamics of prey prehension by chameleons through viscous adhesion. Nat. Phys. 12, 931-935 (2016)

10. Watnick, P. \& Kolter, R. Biofilm, city of microbes. J. Bacteriol. 182, 2675-2679 (2000).

11. Hall-Stoodley, L., Costerton, J. W. \& Stoodley, P. Bacterial biofilms: from the natural environment to infectious diseases. Nat. Rev. Microbiol. 2, 95-108 (2004)

12. Schenk, P. M. et al. Second generation biofuels: high-efficiency microalgae for biodiesel production. BioEnergy Res. 1, 20-43 (2008).

13. Siaut, M. et al. Oil accumulation in the model green alga Chlamydomonas reinhardtii characterization, variability between common laboratory strains and relationship with starch reserves. BMC Biotechnol. 11, 7 (2011).

14. Rüffer, U. \& Nultsch, W. High-speed cinematographic analysis of the movement of Chlamydomonas. Cell Motil. 5, 251-263 (1985).

15. Bloodgood, R. A. in Ciliary and Flagellar Membranes (ed. Bloodgood, R. A.) 91-128 (Springer, 1990).

16. Dufrêne, Y. F. Sticky microbes: forces in microbial cell adhesion. Trends Microbiol. 23, 376-382 (2015).

17. Harris, E. H., Stern, D. B. \& Witman, G. B. The Chlamydomonas Sourcebook 2nd edn (Academic, 2009).

18. Guschina, I. A. \& Harwood, J. L. Lipids and lipid metabolism in eukaryotic algae. Prog. Lipid Res. 45, 160-186 (2006).

19. Hegemann, P. Algal sensory photoreceptors. Annu. Rev. Plant Biol. 59, 167-189 (2008).

20. Bloodgood, R. A. \& Workman, L. J. A flagellar surface glycoprotein mediating cell-substrate interaction in Chlamydomonas. Cell Motil. 4, 77-87 (1984).

21. Rosenbaum, J. L. \& Witman, G. B. Intraflagellar transport. Nat. Rev. Mol. Cell Biol. 3, 813-825 (2002)

22. Laib, J. A., Marin, J. A., Bloodgood, R. A. \& Guilford, W. H. The reciprocal coordination and mechanics of molecular motors in living cells. Proc. Natl Acad. Sci. USA 106, 3190-3195 (2009).
23. Shih, S. M. et al. Intraflagellar transport drives flagellar surface motility. eLife 2, e00744 (2013)

24. Collingridge, P., Brownlee, C. \& Wheeler, G. L. Compartmentalized calcium signaling in cilia regulates intraflagellar transport. Curr. Biol. 23 2311-2318 (2013)

25. Ramaswamy, S. et al. Nanoscale analysis of surface topography and adhesion force measurements of flagella isolated from Chlamydomonas reinhardtii. J. Adv. Microsc. Res. 8, 163-170 (2013).

26. Colbert, M. J., Raegen, A. N., Fradin, C. \& Dalnoki-Veress, K. Adhesion and membrane tension of single vesicles and living cells using a micropipette-based technique. Eur. Phys. J. E 30, 117-121 (2009).

27. Berthold, P. et al. Channelrhodopsin-1 initiates phototaxis and photophobic responses in Chlamydomonas by immediate light-induced depolarization. Plant Cell 20, 1665-1677 (2008).

28. Huang, K. \& Beck, C. F. Phototropin is the blue-light receptor that controls multiple steps in the sexual life cycle of the green alga Chlamydomonas reinhardtii. Proc. Natl Acad. Sci. USA 100, 6269-6274 (2003).

29. Rüffer, U. \& Nultsch, W. Flagellar photoresponses of Chlamydomonas cells held on micropipettes: II. Change in flagellar beat pattern. Cell Motil. Cytoskeleton 18, 269-278 (1991).

30. Bloodgood, R. A. \& May, G. S. Functional modification of the Chlamydomonas flagellar surface. J. Cell Biol. 93, 88-96 (1982).

31. Weibel, D. B. et al. Microoxen: microorganisms to move microscale loads. Proc. Natl Acad. Sci. USA 102, 11963-11967 (2005).

\section{Acknowledgements}

The authors thank M. Lorenz and the Göttingen Algae Culture Collection (SAG) fo providing the Chlamydomonas reinhardtii strain SAG 11-32b. We thank M. Lorenz, K. Dalnoki-Veress and S. Herminghaus for discussions and T. Ostapenko for technical assistance. O.B. acknowledges support from the Joliot ESPCI Paris Chair and the Total-ESPCI Paris Chair.

\section{Author contributions}

O.B. conceived and directed the study. C.T.K. and O.B. designed research. The micropipette force spectroscopy experiments were performed by C.T.K., M.L.B. and C.L.; the micropipette experiments were set up by C.T.K., M.M.M. and O.B. All authors contributed to the discussions. C.T.K. and O.B. wrote the manuscript.

\section{Additional information}

Supplementary information is available in the online version of the paper. Reprints and permissions information is available online at www.nature.com/reprints. Publisher's note: Springer Nature remains neutral with regard to jurisdictional claims in published maps and institutional affiliations. Correspondence and requests for materials should be addressed to O.B.

\section{Competing financial interests}

The authors declare no competing financial interests. 


\section{Methods}

Cell cultivation. Wild-type Chlamydomonas reinhardtii, strain SAG 11-32b, were grown axenically in tris-acetate-phosphate (TAP) medium on a $12 \mathrm{~h}-12 \mathrm{~h}$ day-night cycle in a Memmert IPP 100Plus incubator. The daytime temperature was $24^{\circ} \mathrm{C}$ with a light intensity of $1 \times 10^{20}-2 \times 10^{20}$ photons $\mathrm{m}^{-2} \mathrm{~s}^{-1}$; the night-time temperature was $22^{\circ} \mathrm{C}$ and the light intensity was reduced to zero. Experiments were performed with vegetative cells taken from the cultures in logarithmic growth phase during the daytime on the second to fourth day after incubation. A small amount of the culture, about $0.1-0.2 \mathrm{ml}$, was suspended in the liquid cell to achieve a dilute suspension for experiments in ambient conditions $\left(24-26^{\circ} \mathrm{C}\right)$

Micropipette force spectroscopy. The micropipette force spectroscopy technique is based on a concept that has been put forward to measure dynamic forces in biological systems $\mathrm{s}^{26,32,33}$. This technique relies on the high-resolution optical imaging of the deflection of a double-L-shaped micropipette (see Fig. 1a). We used two inverted microscope set-ups (Olympus IX-73/IX-83) equipped with scientific cameras recording greyscale images at $10 \mathrm{fps}$ (Grashopper, GS3-U3-41C6M-C) to track the deflection of the micropipette, and at approximately $800 \mathrm{fps}$ (PCO, pco.edge 4.2) to visualize the motion of the flagella. Imaging is typically performed at $40 \times$ magnification using long-distance objectives. High-precision motorized linear stages controlled the substrate position (Newport, Conex LTA-HS), while 3-axis piezo-driven manual micromanipulators (Burleigh, PCS-5400) allow for precise adjustments of the micropipette position. The two set-ups were placed on active anti-vibration tables (Accurion, Halcyonics i4-large), both contained in closed boxes to minimize acoustic vibrations and to precisely control the light exposure during the experiment.

We used custom-built liquid cells consisting of two plane-parallel microscope glass slides displaced by O-rings as chamber walls (see Fig. 1b). The liquid cell was filled with approximately $3 \mathrm{ml}$ TAP growth medium to which a small amount of a dilute Chlamydomonas suspension was added from the cell cultures. Experiments were performed with a fully opened condenser aperture to avoid cells preferentially accumulating in the area of interest due to phototaxis and interfering with the experiment. Prior to experiments, the liquid chamber, as well as the O-rings, were cleaned in ethanol. As substrates, we used pieces of a silicon wafer (Si-Mat, silicon wafer: type $\mathrm{P} / \mathrm{Bor}$, orientation $\langle 100\rangle$, resistivity $1-20 \Omega \mathrm{cm}$, unilateral polished) that were attached to a custom-made stainless steel holder and rinsed in ethanol (Merck, Uvasol, Purity 99.9\%) in an ultrasonic bath. For adhesion force measurements on an alternative set of substrates (see Supplementary Fig. 7), we employed hydrophobized substrates by applying a self-assembled monolayer of octadecyltrichlorosilane (OTS), which covalently binds to the oxide layer of the silicon wafer. The substrate preparation followed established recipes ${ }^{34}$.

As force sensors, we use micropipettes made from borosilicate glass capillaries with an initial diameter of $1 \mathrm{~mm}$ (WPI, Borosilicate Glass Capillaries TW100-6). We fabricated micropipettes of several centimetres length with an outer diameter of 10-20 um using a pipette puller (Sutter Instrument, P-97 Flaming/Brown Micropipette Puller). The tips of the micropipettes were cut and eventually fire-polished, resulting in clean straight edges with an opening of approximately $5 \mu \mathrm{m}$. Subsequently, the micropipettes were bent in the characteristic double- $\mathrm{L}$ shape using a microforge (Narishige, microforge MF-900). The long lever arm of a micropipette was approximately $1-3 \mathrm{~cm}$ in length, which was typically two to three orders of magnitude longer than the short nozzle that features the opening to attach the cell. The force sensor was connected to a syringe filled with water to create a slightly negative pressure to hold the Chlamydomonas cell. See Supplementary Fig. 4a for a detailed sketch of the micropipette design.

The deflection of the micropipette from the zero-force position was determined from an auto-correlation analysis of consecutive cross-sectional intensity profiles of the lever position that were extracted from the optical images (see Supplementary Fig. 4b). The auto-correlation analysis is based on a custom-made Matlab algorithm, which picks the maximum of a spline fit to the raw correlation output (see Supplementary Fig. 4c,d). Consequently, the correlation analysis features a sub-pixel deflection resolution of several tens of nanometres, which ultimately leads to a force resolution of a few tens of piconewtons, as discussed in the following section. We define the noise level of the force sensors as the standard deviation of the deflection signal from the equilibrium position with a Chlamydomonas cell being attached to the micropipette. For example, in the experiment displayed in Fig. 3b, the noise level was $23 \mathrm{pN}$, as extracted from data recorded during $200 \mathrm{~s}$ in red-light conditions.

Force sensor calibration. The calibration of the micropipette force sensors was achieved by using the added weight of a variable mass, such as a water droplet in air (see Supplementary Fig. 4b). We recorded the temporal evolution of the volume of the water droplet and simultaneously tracked the resulting deflection of the freely suspended micropipette using a custom-made Matlab algorithm. The force-deflection graph yields a linear relationship and validates that the micropipette force sensor can be treated as a Hookean spring for deflections of up to hundreds of micrometres (see Supplementary Fig. 4e). We obtained the spring constant from the best linear fit to the force-deflection data. The spring constant used to translate the deflection signal into a force measurement in the force spectroscopy experiments was obtained from the mean of multiple independent calibration experiments, yielding a relative error of less than $10 \%$. The spring constants of the force sensors employed in this study varied between $0.2-1 \mathrm{nN} \mu \mathrm{m}^{-1}$, depending on the precise geometrical parameters of the micropipettes. Independent validations of the pipette calibrations were performed in TAP medium and based on the deflection of the micropipettes using a second pre-calibrated sensor (for example, an atomic force microscopy cantilever), confirming the values of the spring constants.

Force-distance spectroscopy. At the beginning of an experiment, the liquid cell was assembled and the Chlamydomonas suspension was injected into the chamber Afterwards, we grasped a Chlamydomonas cell with the micropipette in a manually controlled cell orientation. The viability of the cell was carefully checked at the beginning and at the end of the experiment by monitoring the regular beating of the flagella. To obtain force-distance curves, the raw deflection data of the micropipette were collected based on the image auto-correlation procedure described above and eventually corrected for a linear drift, which is typically less than $2 \mu \mathrm{m}$ during an experiment of $15 \mathrm{~min}$.

Each force-distance curve consists of a substrate-cell approach, a time delay on the substrate, and the retraction of the substrate from the cell (see Supplementary Fig. 5). During the approach and the retraction cycle, the substrate moved at a constant velocity of $1 \mu \mathrm{m} \mathrm{s}^{-1}$, while the substrate stayed at rest for approximately $15 \mathrm{~s}$ in between. This substrate velocity results in a moderate force ramp rate of $0.2-1 \mathrm{nN} \mathrm{s}^{-1}$. Variation of the ramp rate by changing the substrate velocity does not affect the measured adhesion forces (see Supplementary Fig. 6). The dwell time of approximately $15 \mathrm{~s}$ was chosen to ensure that the flagella have accommodated on the substrate in the gliding configuration-that is, straight and in $180^{\circ}$ orientation -in all measurements. The time it takes to achieve this configuration after contact is at most a few seconds, as inferred from top-view optical imaging (see Supplementary Fig. 8). The zero-distance position is defined as the substrate position where the force-distance curve during the approach enters the repulsive regime, corresponding to a positive force acting on the micropipette when the cell body makes contact with the substrate. Note that the distance coordinate directly translates into the pipette deflection since the pipette is orders of magnitudes softer than the attached cell. The maximum pushing force on the cell in contact with the substrate was controlled from one cell to another and ranged from $1-5 \mathrm{nN}$; variation of the pushing force did not affect the adhesion measurement.

The adhesion force data presented in Fig. 1c (inset) comprise independent experiments from 55 different cells, each experiment containing a series of 5 consecutive force-distance curves. The distribution of adhesion forces is fitted by a log-normal distribution, $f(x)=1 /(\sqrt{2 \pi} \alpha x) \exp \left(-(\ln (x)-\beta)^{2} / 2 \alpha^{2}\right)$, with $\alpha=0.82$ and $\beta=1.0$ as best fit parameters. Experimental data shown in the inset of Fig. $2 \mathrm{a}$ include independent experiments from 17 different cells, each experiment containing 10 force-distance curves in white and 10 force-distance curves in red light. All individual data points displayed in Figs $2 \mathrm{~b}-\mathrm{f}$ and 4 and Supplementary Figs 2a, 3 and 6 comprise a series of 5 force-distance curves each.

Light conditions. The light conditions were carefully controlled during all adhesion experiments. White-light force-distance experiments were performed at approximately $5 \times 10^{20}$ photons $\mathrm{m}^{-2} \mathrm{~s}^{-1}$ standard microscopy illumination (Olympus, Halogen light bulb JC 12V100W). Red-light conditions of the same light intensity were realized using a bandpass filter $(665 \mathrm{~nm}$, full-width at half-maximum (FWHM) $65 \mathrm{~nm}$ ) in the experiment shown in Fig. 2b and a high-pass filter $(\geq 550 \mathrm{~nm})$ in the experiment shown in Fig. 2c. Otherwise, a bandpass interference filter $(671 \mathrm{~nm}$, FWHM $10 \mathrm{~nm})$ at approximately $5 \times 10^{19}$ photons $\mathrm{m}^{-2} \mathrm{~s}^{-1}$ was employed. For experiments that directly compare different wavelengths, as shown in Fig. 2d, we exposed the cells to $3 \times 10^{19}$ photons $\mathrm{m}^{-2} \mathrm{~s}^{-1}$ intensity (Cool-LED, Olympus) using a series of narrow-band interference filters in addition to a red bandpass interference filter $(671 \mathrm{~nm}$, FWHM $10 \mathrm{~nm}$ ): $400 \mathrm{~nm}$ (FWHM $5 \mathrm{~nm}$ ), $470 \mathrm{~nm}$ (FWHM $5 \mathrm{~nm}$ ), $554 \mathrm{~nm}$ (FWHM $25 \mathrm{~nm}$ ), $641 \mathrm{~nm}$ (FWHM $18 \mathrm{~nm}$ ). The adhesion force distribution in blue light shown in Fig. 2e and the intensity-dependent adhesion measurements shown in Fig. $2 \mathrm{f}$ were obtained by using the red bandpass interference filter (671 nm, FWHM $10 \mathrm{~nm}$ ) and additionally exposing the cells to blue light ( $470 \mathrm{~nm}$, FWHM: $5 \mathrm{~nm}$, Cool-LED, Olympus). The intensity is adapted by applying optical density filters. The intensity was calibrated using a power meter (Thorlabs, PM100D, sensor: S130C) and converted into a photon density flux (photons $\mathrm{m}^{-2} \mathrm{~s}^{-1}$ ) by optically determining the spatial extent of the illuminated area.

The auto-adhesion experiments were performed in red-light conditions at approximately $5 \times 10^{19}$ photons $\mathrm{m}^{-2} \mathrm{~s}^{-1}$ (bandpass interference filter: $671 \mathrm{~nm}$, FWHM $10 \mathrm{~nm}$ ) and in white illumination using a white-light LED (type P585E, $12 \mathrm{~V} / \mathrm{DC}$ luminous intensity $6800 \mathrm{mcd}$ ) at approximately $2 \times 10^{19}$ photons $\mathrm{m}^{-2} \mathrm{~s}^{-1}$ as an additional light source. 
If not stated otherwise, we exposed the cells at least one hour to white-light conditions to ensure that experiments were performed after the adhesion force had saturated (see Supplementary Fig. 3b).

Auto-adhesion experiments. Auto-adhesion experiments required the same preparations as mentioned above. Here, the substrate position remained fixed during the entire experiment, while the cell was brought in close proximity to the substrate (approximately $4-8 \mu \mathrm{m}$ distance between the substrate and the cell body). Immediately after turning on the white-light LED, the flagella beating pattern changed from breaststroke to an undulatory motion for hundreds of milliseconds, in accordance with previous studies relating this effect to a photophobic shock ${ }^{35-37}$. In all cases, the beating recovered the normal breaststroke pattern afterwards. The delay time related to the auto-adhesion is defined as the time interval between turning on the white light and the first response as seen in the micropipette deflection signal. The statistical distribution of delay times was obtained from 17 different cells, each cell contributing 5 individual auto-adhesion events.

Proteolysis experiments. In the proteolysis experiments, we recorded the adhesion force of several individual cells and, subsequently, added pronase (Roche Diagnostic) to the TAP medium in the liquid chamber at a concentration of $0.1 \mathrm{mg} \mathrm{ml}^{-1}$. Note that the cell was held by the micropipette in solution and not in contact with the substrate during pronase treatment. During the treatment in white light, we performed adhesion experiments every $15 \mathrm{~min}$. After the exposure of the cells to pronase in red light for two hours, the adhesion force was determined immediately after turning on the white-light illumination without flushing the liquid cell with fresh TAP medium.

Data availability. The data that support the plots within this paper and other findings of this study are available from the corresponding author upon request.

\section{References}

32. Francis, G. W., Fisher, L. R., Gamble, R. A. \& Gingell, D. Direct measurement of cell detachment force on single cells using a new electromechanical method. J. Cell Sci. 87, 519-523 (1987).

33. Yeung, A. K. C. \& Pelton, R. Micromechanics: a new approach to studying the strength and breakup of flocs. J. Colloid Interface Sci. 184, 579-585 (1996).

34. Lessel, M. et al. Self-assembled silane monolayers: an efficient step-by-step recipe for high-quality, low energy surfaces. Surf. Interfaces Anal. 47, 557-564 (2015)

35. Schmidt, J. A. \& Eckert, R. Calcium couples flagellar reversal to photostimulation in Chlamydomonas reinhardtii. Nature 262, 713-715 (1976).

36. Brokaw, C. J., Luck, D. J. \& Huang, B. Analysis of the movement of Chlamydomonas flagella: the function of the radial-spoke system is revealed by comparison of wild-type and mutant flagella. J. Cell Biol. 92, 722-732 (1982).

37. Rüffer, U. \& Nultsch, W. Flagellar photoresponses of Chlamydomonas cells held on micropipettes: III. shock response. Bot. Acta 108, 255-265 (1995). 